\title{
A Retrospective Analysis on the Clinical Efficiency of Dual Trigger During Vitro Fertilization and Embryo Transfer (IVF-ET) Treatment for Women with Diminished Ovarian Reserve
}

\section{Ling Zhang}

Zhejiang Provincial People's Hospital

\section{Li-mei Wu}

Zhejiang Provincial People's Hospital

Qiong-xiao Huang

Zhejiang Provincial People's Hospital

Meng-xia Ji

Zhejiang Provincial People's Hospital

Shi-shi Li

Zhejiang Provincial People's Hospital

Lin Zhang

Zhejiang Provincial People's Hospital

Zhen Jin

Zhejiang Provincial People's Hospital

Yi-er Zhou

Zhejiang Provincial People's Hospital

\section{Chong-yi Shu}

Zhejiang Provincial People's Hospital

Jing Shu ( $\sim$ shujing@hmc.edu.cn )

Zhejiang Provincial People's Hospital

Wei-hai Xu

Zhejiang Provincial People's Hospital

\section{Research Article}

Keywords: Dual trigger, Diminished ovarian reserve, IVF-ET

Posted Date: January 25th, 2022

DOI: https://doi.org/10.21203/rs.3.rs-1258868/v1 
License: (c) (i) This work is licensed under a Creative Commons Attribution 4.0 International License. Read Full License 


\section{Abstract}

Background: Due to poor number and quality of oocytes, the reproductive prognosis of diminished ovarian reserve (DOR) patients is obstinately disapproving. This study will compare the effects of double trigger and hCG trigger on the culture outcome and implantation outcome during the IVF treatment for DOR patients.

Methods: a total of 857 controlled ovarian stimulation (COS) cycles and 366 frozen embryo transfer (FET) cycles were included in this study. The difference in retrieval cancellation, oocyte retrieval, number of oocytes, maturation oocytes, fertilization, embryos were evaluated between the two groups in the COS dataset, while implantation, clinical pregnancy, ongoing pregnancy, and spontaneous abortion were analyzed based on FET dataset. To robustly evaluate the marginal effect of the study factor as well as other covariates, a cluster-weighted GEE model was exploited to fit the uniqueness of IVF-ET data.

Results: Neither the retrieval outcome, nor cultural or implantation outcomes were improved after dualtrigger. The OR values were 1.08 (95\% Cl: $0.41 ~ 2.78)$ for retrieval cancellation, 1.33 (95\% Cl: $0.89 \sim 2.00$ ) for oocyte harvest, 0.99 (95\%: 0.97 1.02) for oocyte maturation, 1.03 (95\% Cl: 0.94 1.12) for fertilization, 1.04 (95\%Cl: $0.94 ~ 1.15$ ) for viable embryo, and 1.03 (95\%Cl: $0.88 \sim 1.19$ ) for top quality embryo. As to the transfer outcome, no difference was identified between the two groups, with the ORs 0.90 (95\% Cl: $0.62 ~ 1.30$ ) for implantation and 0.97 (95\%Cl: $0.56 \sim 1.69$ ) for clinical pregnancy. This insignificance remained unchanged after adjusting for the covariates such as age, BMI, infertility duration, etc.

Conclusions: Dual triggering the final oocyte maturation combined of GnRH-a and standard dose of hCG cannot significantly improve the retrieval, cultural or implantation outcomes of IVF-ET treatments in women with DOR.

\section{Background}

Decreased ovarian reserve (DOR) hasbeen posingstubborn challenges to most reproductive clinicians in recent years.Even worse, this problem is exacerbated by the delayed reproductive age in human.Inconventionalin vitro fertilization and embryo transfer(IVF-ET) practice, the outcome of patients with DOR is unsurprisingly suboptimal due tomeasly follicles, poor oocyte quality, and subsequently limited number of transferable embryos. To this end,certain modifications have been attempted to different aspects of IVF-ET treatment in order to gain a better outcome, including pretreatment with various drugs, COS protocols as well as various supplements [1]. However, most of the measures had limited success, raising the necessity that additional modification be investigated.

Trigger of the final maturation of oocyte is one of the most key aspects duringIVF-ET procedure. Human chorionic gonadotropin ( $\mathrm{hCG}$ ) and gonadotropin releasing hormone agonist ( $\mathrm{GnRHa}$ ) are two commonly used tools trigger. The former actsas a trigger by binding to luteinizing hormone (LH) receptorand mimicking the biological role of LHbased on structural and biological similarities,[2], and the latter by 
promoting the pulsed release of endogenous $\mathrm{LH}$ andfollicle-stimulating hormone (FSH)through binding to pituitary $\mathrm{GnRH}$ receptor $[3,4]$. Three trigger protocols have been developedbased on the two drugs, including hCG alone (hCG trigger), GnRHaalone (GnRHa trigger) and GnRHa plus reduced dose of hCG (dual trigger)[5]. It is generally acknowledged that harvesting enough mature oocytes and transferable embryos is one decisive factor for the success of IVF-ET treatment, underlining the importance of trigger protocols, especially for DOR patients.

A bulk of evidence have suggested that dual trigger haveseveralsignificant advantages over hCG or GnRHa protocol,such as better luteal phase supportand lower risk of OHSSin normal and/or high respond patients[6-8]. More importantly, some reports showed that dual trigger may improve oocyte retrieval and maturation, cultural outcome, as well as embryo implantation in subjects with normal ovarian reservation[9, 10]. In view of this, the application of dual trigger to DOR patientshas been proposed, especiallyfor itspotential in promisingnumber and viability of embryos[11-14]. However, current conclusions remainedinconsistent, with some studies believing that double trigger can significantly improve the rate of fertilized eggs, viable embryos, or embryo implantation[11, 12], while another two studies failing to identifyany significance in these endpoints $[13,14]$.Moreover, pitifully few evidence has been available for the improvement of oocyte retrieval and maturation rate bydual trigger[14].Thus, more researches are needed to comprehensively evaluate the clinical efficiency of dual trigger in DOR.

Additionally, one of the biggest challenges in discriminatingthe potential factors that influencing clinical outcomes of IVF-ET treatment is toseek a suitable statistical model for the data with evident cluster dependence such as repeated cycles, as well as toproperly estimate marginal effectsof each potential covariateunder the conditions that the clinical outcome is influenced by multiple factors.In this retrospective analysis,we carried out a clusterweighted generalized estimating equation (WGGE) to evaluate the contribution of trigger protocol to the outcomes of IVF-ET treatmentin DOR patients.

\section{Materials And Methods}

\section{Study design and participants}

This is a retrospective analysis on the IVF-ET cyclesfrom January, 2016 to August, 2021 atthe Reproductive Center of Zhejiang Provincial People's Hospital, China. The study protocol was approved by the Ethics Committee of Zhejiang Provincial People's Hospital.

Decreased ovarian reserve (DOR) was defined according the POSEIDON criteria, and fulfilled both following criteria:anti-Mullerian hormone $(\mathrm{AMH})<1.2 \mathrm{ng} / \mathrm{ml}$ and antral follicle count [AFC ( $\mathrm{n})$ ] $<5$. IVF cycles with controlled ovarian stimulation GnRH antagonist, PPOS or mild stimulation protocols were included. Exclusion criteria were those with chromosome abnormality (carrier of balanced translocation or inversion), chemotherapy or radiotherapy treatment, $\mathrm{BMI}>30 \mathrm{~kg} / \mathrm{m}^{2}$, or basic $\mathrm{FSH}>20 \mathrm{IU} / \mathrm{L}$. After the final recruitment by the inclusion and exclusion criteria, a total of 381 patients, who finished 857 cos cycles, were selected into present study, and divided into hCGtrigger and dual trigger groups. 


\section{Controlled ovarian stimulation and oocyte maturation trigger}

For mild stimulation protocol, clomiphene citrate (CODAL SYNTO LTD., Cyprus) was administrated fromcycle day 2 at a daily dose of 50-100 mg combined with 75-150U human menopausal gonadotropin (hMG)(Lizhu Pharmaceutical, China). From cycle day 8, GnRH-antagonist (Cetrorelix, Merck Serono, Germany) was added when serum LH exceeded the level of $10 \mathrm{IU} / \mathrm{mL}$.For progestin-primed ovarian stimulation (PPOS) protocol, medroxyprogesterone acetate (MPA) (Zhejiang Xianju Pharmaceutical Co., China) at a daily dose of 6-8 $\mathrm{mg}$ and $\mathrm{hMG}$ at 75 to $225 \mathrm{IU}$ per day were given from cycle day 2 until the trigger day.For $\mathrm{GnRH}$-antagonist regimen, gonadotropins including recombinant follicle stimulating hormone (Gonal-f; Merck Serono, Germany) or hMG (Lizhu Pharmaceutical Factory, China) were administratedin a flexible way fromcycleday 2 at a dose of 100-300U per day, and adjusted according to ovarian response. When the diameter of leading follicles reached $12 \mathrm{~mm}$, a daily dose of $0.25 \mathrm{mg} \mathrm{GnRH}$ antagonist was injected subcutaneously. When at least one follicle diameter $18 \mathrm{~mm}$ or two follicles diameter $17 \mathrm{~mm}$, final oocytematuration was triggered by either recombined hCG (0.25mg;Merck Serono, Germany) alone, or combination of hCGwith 0.1 mg triptorelin (Decapeptyl, Ferring Pharmaceuticals).

\section{Embryo culture and score}

Atabout 36 hours after oocyte trigger, the cumulus-oocyte complexes (COCs)were aspired by transvaginal puncture under ultrasound guidance.Depending on the semen quality or previous IVF history, the obtained oocytes were inseminated by conventional IVF or intracytoplasmic sperm injection (ICSI). Embryos at day 3 were graded by theblastomere symmetry and fragmentation according to the Istanbul consensus on embryo assessment[15]. Day 3 embryos graded as 'good'with blastomere number were defined as top-quality embryos, and directly cryopreserved by vitrification,while rest non-top-quality embryos underwent a continuous blastocyte culture. Onlyviable blastocystswithinner cell mass or trophectoderm gradedabove 'B' according to Gardner score system were cryopreservedon day 5 or day $6[16]$. All vitrification and warming procedures were conducted according to the instructions of reagent manufacturer (Vitrolife, Sweden).

\section{Endometrium preparation, frozen embryo transfer (FET)}

Either a natural cycle, hormone replacement (HRT) cycle, or induced ovulation cycle was used for endometrium preparation. Briefly, for natural cycles, progesterone treatment started from the ovulation day or 2 days after LH surge.For HRT cycle, oralestradiol (estradiol valerate, Delpharm Lille S.A.S, France) was administered at $6 \mathrm{mg} /$ day from cycle day 3 onwards, and atday 7 after HRT initiation, $5 \mathrm{~g} / \mathrm{day}$ estradiol gels (Besins Manufacturing Belgium)wereadded for another 5-7 days if endometrial lining $<7 \mathrm{~mm}$. Once the endometrial reached a satisfactory thickness, $40 \mathrm{mg}$ progesterone was injected.A maximum of two embryos were thawed on the morning of thetransfer day, and transferred under ultrasound guidance. Once pregnancy was achieved, the progesterone and estradiol supplementation were continued until 10 weeks of gestation.

\section{Outcome measures}


The primary endpoints of present study included retrieval cancel rate, cycle rate of oocyte harvesting, oocyte harvesting rate, oocyte maturation rate, viable embryo rate and implantation rate. Here, retrieval cancel rate was defined as the number of cycles cancelled due to premature ovulation divided by the number of total cycles, cycle rate of oocyte harvestingasthe number of cycles with successful oocyte harvesting divided by the number of oocyte pick-up(OPU) cycles,oocyte harvesting rate as the number of total harvested oocytes divided by the number of follicles aspirated (diameter $12 \mathrm{~mm}$ ) on theOPU day, oocyte maturation and viable embryo rate as the ratio of MII oocytenumber and viable embryo to total number of collected oocytes, respectively, and the implantation rate as the number of gestational sacs visualized under ultrasound examination divided by the number of embryos transferred.The secondary endpoints were clinical pregnancy rateandearly miscarriage rate.A clinical pregnancy referred toa gestational sac under ultrasound, with or without heartbeat, and early miscarriage rate to the spontaneous loss of a clinical pregnancy before 12th weeks ofgestation.

\section{Statistical analysis}

The data were analyzed by SPSS statistical software (version 21.0, IBM Corp., USA). The continuousvariableswere expressed as mean \pm standard deviation ( $\pm S D)$, and the category one as $n(\%)$. The comparison betweenthe two groups was conducted withstudent $t$ test or Fisher's exact test. To identify the genuine association of trigger protocols with oocyte retrieval, culture outcomes and implantation endpoints, a cluster-weighted generalized estimating equation (GEE) models with a log link were used to account for within person correlations between repeated cycles in presence of nonignorable cluster size $[17,18]$.During GEE models,a binomial distribution with log link function were specified for retrieval cancel rate, cycle rate of oocyte harvesting, clinical and ongoing pregnancy, and a poisson distribution with log link function for oocyte harvesting rate, culture outcomes and implantation rate.In poisson distribution models, a logarithm of the number of punctured follicles or transferred embryos were taken as the offset.The inverse of the number of oocytes or repeated cycles was taken as the weighting coefficient. Results were presented as a weighted average over levels of categorical variables according to their relative frequency. Statistical significance was defined as $P<0.05$.

\section{Results}

Present study included a total of 381 patients, who finished857COS cycles. Of all included cycles, 21 canceled before oocyte retrieval due to premature ovulation, and 124 failed to harvest oocytes. Thus, 836 cycles fulfilled oocyte pick-up, and733 cycles underwentin vitro culture procedure. The baseline characteristics for the patients and cycles included were summarized in Table 1 and 2, respectively. There was no significant difference in age, $\mathrm{BMI}, \mathrm{AMH}$, basic serum $\mathrm{FSH}$, infertility durationorinfertility diagnosis, whereas a significance existed in COS protocol $(p=0.0004)$, total dose of gonadotrophins $(p<0.0001)$, and days of gonadotrophins administration $(p<0.0001)$.

\section{Table 1. Baseline characteristics of included couples}




\begin{tabular}{|ll|}
\hline Parameter & Values \\
\hline No. of couples $(\mathrm{n})$ & 381 \\
\hline Female age (yrs) & $37.94 \pm 6.14$ \\
\hline BMI $(\mathrm{Kg} / \mathrm{m} 2)$ & $22.10 \pm 2.90$ \\
\hline AMH $(\mathrm{ng} / \mathrm{mL})$ & $0.50 \pm 0.33$ \\
\hline Basic FSH (IU/L) & $9.27 \pm 4.89$ \\
\hline Antral follicle count (AFC, $\mathrm{n})$ & $2.56 \pm 1.09$ \\
\hline Infertility duration (yrs) & $3.72 \pm 3.44$ \\
\hline Primary infertility, $\mathrm{n}(\%)$ & $113(29.66)$ \\
\hline Male factor, $\mathrm{n}(\%)$ & $166(43.57)$ \\
\hline Endometriosis, $\mathrm{n}(\%)$ & $47(12.34)$ \\
\hline Tubal factor, $\mathrm{n}(\%)$ & $56(14.70)$ \\
\hline Ovulation disorder, $\mathrm{n}(\%)$ & $29(7.61)$ \\
\hline Unexplained, $\mathrm{n}(\%)$ & $25(6.56)$ \\
\hline
\end{tabular}

Table 2. Baseline and treatment characteristics of studied subjects. 


\begin{tabular}{|c|c|c|c|c|}
\hline & Total $(n=857)$ & hCG (n=258) & Dual trigger $(n=599)$ & $P$ values \\
\hline Female age (yrs) & $38.62 \pm 6.25$ & $38.38 \pm 6.48$ & $38.72 \pm 6.15$ & 0.454 \\
\hline $\mathrm{BMI}\left(\mathrm{Kg} / \mathrm{m}^{2}\right)$ & $22.01 \pm 2.75$ & $21.88 \pm 2.78$ & $22.06 \pm 2.74$ & 0.388 \\
\hline $\mathrm{AMH}(\mathrm{ng} / \mathrm{mL})$ & $0.47 \pm 0.32$ & $0.46 \pm 0.34$ & $0.47 \pm 0.31$ & 0.655 \\
\hline Basic FSH(IU/L) & $9.45 \pm 5.09$ & $9.14 \pm 5.08$ & $9.59 \pm 5.1$ & 0.233 \\
\hline $\operatorname{AFC}(n)$ & $2.54 \pm 1.08$ & $2.44 \pm 1.04$ & $2.58 \pm 1.09$ & 0.092 \\
\hline Infertility duration (yrs) & $4.06 \pm 3.89$ & $3.69 \pm 3.26$ & $4.22 \pm 4.12$ & 0.065 \\
\hline Gn dose (IU) & $1334.89 \pm 789.14$ & $1063.59 \pm 739$ & $1448.95 \pm 782.24$ & $<.0001$ \\
\hline Days of Gn (d) & $8.56 \pm 3.38$ & $7.53 \pm 3.49$ & $9 \pm 3.24$ & $<.0001$ \\
\hline Primary infertility, n(\%) & $253(29.52)$ & $72(27.91)$ & $181(30.22)$ & 0.497 \\
\hline \multicolumn{5}{|c|}{ Controlled ovarian stimulating protocol } \\
\hline PPOS, n(\%) & $329(38.39)$ & $84(32.56)$ & $245(40.90)$ & 0.0004 \\
\hline Mini-stimulation, n(\%) & $355(41.42)$ & $101(39.15)$ & $254(42.40)$ & \\
\hline GnRH-ant, n(\%) & $139(16.22)$ & $55(21.32)$ & $84(14.02)$ & \\
\hline Others, $\mathrm{n}(\%)$ & $34(3.97)$ & $18(6.98)$ & $16(2.67)$ & \\
\hline \multicolumn{5}{|l|}{ Infertility diagnosis, n (\%) } \\
\hline Male factor & $382(44.92)$ & $115(44.57)$ & $270(45.08)$ & 0.892 \\
\hline Endometriosis & $96(11.20)$ & $34(13.18)$ & $62(10.35)$ & 0.229 \\
\hline Ovulation disorder & $55(6.42)$ & $19(7.36)$ & $36(6.01)$ & 0.458 \\
\hline Tubal factor & $127(14.82)$ & $40(15.50)$ & $87(14.52)$ & 0.711 \\
\hline Unexplained & $59(6.88)$ & $20(7.75)$ & $39(6.51)$ & 0.510 \\
\hline Retrieval cancel, $\mathrm{n}(\%)$ & $21(2.45)$ & $6(2.33)$ & $15(2.50)$ & 0.877 \\
\hline Oocyte not harvestcycle, n(\%) & $124(14.47)$ & $44(17.05)$ & $80(13.36)$ & 0.158 \\
\hline ICSI, n (\%) & $265(36.15)$ & $76(35.51)$ & $189(36.42)$ & 0.909 \\
\hline
\end{tabular}

$\mathrm{BMI}$, body mass index; $\mathrm{AMH}$, anti-Mullerian hormone; AFC, antral follicle count; PPOS, progestin primed ovarian stimulation protocol; ICSI, Intracytoplasmic sperm injection.

*P value was calculated based on student $t$ test for continuous variable and Fisher exact test for categorical variables. 
The mean number of punctured follicles $(2.18 \pm 1.49$ and $2.31 \pm 1.41)$, retrieved oocytes $(1.86 \pm 1.21$ and $1.94 \pm 1.16)$, MIl oocytes ( $1.79 \pm 1.18$ and $1.85 \pm 1.18)$, fertilized eggs $(1.21 \pm 1.08$ and $1.29 \pm 1.04)$, viable embryos (1.10 \pm 1.07 and $1.20 \pm 1.01)$ and top-quality embryos $(0.75 \pm 0.94$ and $0.8 \pm 0.87)$ were slightly higher in the dual trigger groups as compared with the hCG, but this difference did not reach a statistical significance (Table.3).To ascertain the robustness of our results, we also evaluated the difference in therelative valueof above endpoints between the two groups, anddraw aconsistent conclusion (Table 3). Furtherly, we employeda cluster-weighted GEE model, a statistical methodcompatible with repeated measurement data and count data, tocalculate the edge effect of studied variablewith or without adjusting for confounding factors such as age, BMI, primary infertility, COS protocols et al.Our robust analysis did not showany superior or inferior influence on oocyte retrieval or in vitro culture outcomes for dual trigger as related to HCG group, which was coincided withabove result(Table 4).

Table 3. Outcomes of oocyte retrieving, in vitro fertilization and embryo culture.

\begin{tabular}{|lllll|}
\hline & $\begin{array}{l}\text { Total } \\
(\mathbf{n = 7 3 3 )}\end{array}$ & $\begin{array}{l}\text { hCG } \\
(\mathbf{n = 2 1 4})\end{array}$ & $\begin{array}{l}\text { Dual trigger } \\
(\mathbf{n = 5 1 9 )}\end{array}$ & P value \\
\hline No. of follicles punctured per cycle & $2.27 \pm 1.43$ & $2.18 \pm 1.49$ & $2.31 \pm 1.41$ & 0.271 \\
\hline Oocyte retrieved, $\mathrm{n}(\%)$ & $1595(83.99)$ & $399(85.44)$ & $1007(83.99)$ & 0.463 \\
\hline No. of oocytes retrieved per cycle & $1.92 \pm 1.18$ & $1.86 \pm 1.21$ & $1.94 \pm 1.16$ & 0.429 \\
\hline Mature oocytes, $\mathrm{n}(\%)$ & $1507(94.48)$ & $382(95.74)$ & $953(94.64)$ & 0.475 \\
\hline No. of mature oocyte per cycle & $1.83 \pm 1.18$ & $1.79 \pm 1.18$ & $1.85 \pm 1.18$ & 0.009 \\
\hline 2PN eggs, $\mathrm{n}(\%)$ & $1044(65.45)$ & $258(64.66)$ & $670(66.53)$ & 0.722 \\
\hline No. of 2PN eggs per cycle & $1.27 \pm 1.05$ & $1.21 \pm 1.08$ & $1.29 \pm 1.04$ & 0.316 \\
\hline Viable embryos, $\mathrm{n}(\%)$ & $957(60.00)$ & $236(59.15)$ & $621(61.67)$ & 0.382 \\
\hline No. of viable embryos per cycle & $1.17 \pm 1.03$ & $1.10 \pm 1.07$ & $1.20 \pm 1.01$ & 0.262 \\
\hline Top-quality embryo, $\mathrm{n}(\%)$ & $643(40.31)$ & $160(40.10)$ & $415(41.21)$ & 0.702 \\
\hline $\begin{array}{l}\text { No. of top-quality embryos per } \\
\text { cycle }\end{array}$ & $0.78 \pm 0.89$ & $0.75 \pm 0.94$ & $0.80 \pm 0.87$ & 0.474 \\
\hline
\end{tabular}

*P value was calculated with one-way ANOVA test for continuous variables, and Fisher exact test for categorical variables. $2 \mathrm{PN}$, two pronucleus.

Table 4. Weighted GEE analysis on the effect of trigger protocol on the endpoint of oocyte retrieving, in vitro fertilization and embryo culture. 


\begin{tabular}{|c|c|c|c|c|c|c|}
\hline & \multicolumn{3}{|c|}{ Model 1} & \multicolumn{3}{|c|}{ Model 2} \\
\hline & ORs & $95 \% \mathrm{Cl}$ & $\begin{array}{l}P \\
\text { value }\end{array}$ & ORs & $95 \% \mathrm{Cl}$ & $\begin{array}{l}P \\
\text { value }\end{array}$ \\
\hline Retrieval cancellation & 1.08 & $\begin{array}{l}0.41 \\
\sim 2.78\end{array}$ & 0.877 & 1.1 & $\begin{array}{l}0.40 \\
\sim 3.03\end{array}$ & 0.851 \\
\hline Cycle rate of oocyte harvest & 1.33 & $\begin{array}{l}0.89 \\
\sim 2.00\end{array}$ & 0.159 & 1.32 & $\begin{array}{l}0.87 \\
\sim 2.00\end{array}$ & 0.195 \\
\hline Oocyte harvest per follicle & 1.01 & $\begin{array}{l}0.95 \\
\sim 1.06\end{array}$ & 0.829 & 1.01 & $\begin{array}{l}0.95 \\
\sim 1.06\end{array}$ & 0.789 \\
\hline $\begin{array}{l}\text { No. of mature oocytes per retrieved } \\
\text { oocyte }\end{array}$ & 0.99 & $\begin{array}{l}0.97 \\
\sim 1.02\end{array}$ & 0.567 & 0.99 & $\begin{array}{l}0.96 \\
\sim 1.01\end{array}$ & 0.359 \\
\hline No. of $2 \mathrm{PN}$ eggs per retrieved oocyte & 1.03 & $\begin{array}{l}0.94 \\
\sim 1.12\end{array}$ & 0.52 & $1.02 \dagger$ & $\begin{array}{l}0.93 \\
\sim 1.11\end{array}$ & 0.623 \\
\hline No. of viable embryo per retrieved oocyte & 1.04 & $\begin{array}{l}0.94 \\
\sim 1.15\end{array}$ & 0.411 & $1.03+$ & $\begin{array}{l}0.93 \\
\sim 1.14\end{array}$ & 0.548 \\
\hline $\begin{array}{l}\text { No. of top-quality embryo per retrieved } \\
\text { oocyte }\end{array}$ & 1.03 & $\begin{array}{l}0.88 \\
\sim 1.19\end{array}$ & 0.719 & $1.02 \dagger$ & $\begin{array}{l}0.88 \\
\sim 1.19\end{array}$ & 0.814 \\
\hline
\end{tabular}

Effect of trigger protocol was evaluated as risk ratio $(95 \% \mathrm{Cl})$ with dual trigger protocol as the reference.

Model1, weighted GEE analysis model for data from repeated cycles; model2, weighted GEE analysis adjusted forfemale age, infertility diagnosis, infertility duration, primary infertility, AMH, basic FSH and AFC.

† Additional adjusted for male factor and ICSI procedure.

A total of 366 FET cycles were included into the transfer dataset for evaluating the influence of trigger protocols on implantation outcomes. There was no significant difference between the two groups in terms of baseline characteristics, such as women's age, BMl, endometriumthickness as well as the number of transferred embryos (Table 5). Our results showed that transferring the embryos from hCG trigger might produce higherrates of implantation(26.09\% and 22.62\%), clinical pregnancy (32.35\% and $29.17 \%)$ and ongoing pregnancy $(23.53 \%$ and $18.56 \%)$, while lower early miscarriage $(24.24 \%$ and $35.06 \%)$ as compared to those in the dual trigger group, however, neither univariate Chi square test nor robust cluster-weighted GEE model identified a statistical significance (Table 5 and 6 ).

\section{Table 5区 Characteristics and outcomes of embryo transfer cycles}




\begin{tabular}{|c|c|c|c|c|}
\hline & Total $(n=366)$ & hCG $(n=102)$ & Dual trigger $(n=264)$ & $P$ value \\
\hline Female age (yrs) & $38.64 \pm 5.84$ & $37.95 \pm 6.78$ & $38.91 \pm 5.42$ & 0.160 \\
\hline $\mathrm{BMI}(\mathrm{Kg} / \mathrm{m} 2)$ & $21.91 \pm 2.75$ & $22.07 \pm 2.74$ & $21.85 \pm 2.75$ & 0.495 \\
\hline $\mathrm{AMH}(\mathrm{ng} / \mathrm{mL})$ & $0.55 \pm 0.32$ & $0.53 \pm 0.35$ & $0.56 \pm 0.31$ & 0.403 \\
\hline Basic FSH (IU/L) & $8.17 \pm 3.93$ & $8.05 \pm 4.31$ & $8.22 \pm 3.78$ & 0.719 \\
\hline $\operatorname{AFC}(n)$ & $2.75 \pm 1.09$ & $2.78 \pm 1.06$ & $2.75 \pm 1.09$ & 0.763 \\
\hline Infertility duration (yrs) & $4.10 \pm 3.84$ & $3.84 \pm 3.59$ & $4.2 \pm 3.94$ & 0.428 \\
\hline No. of embryos transferred & $1.44 \pm 0.50$ & $1.35 \pm 0.48$ & $1.47 \pm 0.51$ & 0.040 \\
\hline No. of top embryos transferred & $1.10 \pm 0.64$ & $1.02 \pm 0.6$ & $1.14 \pm 0.65$ & 0.116 \\
\hline Endometrial thickness (mm) & $9.41 \pm 2.46$ & $9.41 \pm 2.46$ & $9.67 \pm 2.36$ & 0.358 \\
\hline Male factor, $n(\%)$ & $167(45.63)$ & $48(47.06)$ & $119(45.08)$ & 0.733 \\
\hline Unexplained factor, $\mathrm{n}(\%)$ & $30(8.20)$ & $9(8.82)$ & $21(7.95)$ & 0.786 \\
\hline \multicolumn{5}{|l|}{ Controlled ovarian stimulation } \\
\hline PPOS, n(\%) & $126(34.43)$ & $28(27.45)$ & $98(37.12)$ & 0.055 \\
\hline Mini-stimulation, $\mathrm{n}(\%)$ & $171(46.72)$ & $47(46.08)$ & $124(46.97)$ & \\
\hline GnRH-ant, n(\%) & $53(14.48)$ & $19(18.63)$ & $34(12.88)$ & \\
\hline Others, $\mathrm{n}(\%)$ & $16(4.37)$ & $8(7.84)$ & $8(3.03)$ & \\
\hline ICSI, n(\%) & $127(34.70)$ & $34(33.33)$ & $93(35.23)$ & 0.771 \\
\hline Embryo implantation, $n(\%)$ & $124(20.60)$ & $36(26.09)$ & $88(22.62)$ & 0.410 \\
\hline Clinical pregnancy, n(\%) & $110(30.05)$ & 33 (32.35) & $77(29.17)$ & 0.551 \\
\hline ongoing pregnancy, $\mathrm{n}(\%)$ & 73 (19.95) & $24(23.53)$ & $49(18.56)$ & 0.286 \\
\hline Early miscarriage, $\mathrm{n}(\%)$ & $35(31.82)$ & $8(24.24)$ & $27(35.06)$ & 0.264 \\
\hline Ectopic Pregnancy, n(\%) & $2(1.82)$ & $1(3.03)$ & $1(1.30)$ & 0.551 \\
\hline
\end{tabular}

*P value was calculated based on one-way ANOVA test for continuous variable and Fisher exact test for categorical variables.

Table 6 Weighted GEE analysis on the effect of trigger protocol on clinical outcomes after embryo transfer 


\begin{tabular}{|lllllll|}
\hline & \multicolumn{7}{c|}{ Model 1 } & \multicolumn{5}{c|}{ Model 2 } \\
\cline { 2 - 7 } & OR value & $95 \% \mathrm{Cl}$ & P value & OR value & $95 \% \mathrm{Cl}$ & P value \\
\hline Clinical pregnancy & 0.97 & $0.56 \sim 1.69$ & 0.911 & 1.12 & $0.68 \sim 1.85$ & 0.636 \\
\hline Implantation $\ddagger$ & 0.90 & $0.62 \sim 1.30$ & 0.576 & 1.00 & $0.71 \sim 1.41$ & 0.993 \\
\hline Early miscarriage & 1.64 & $0.53 \sim 5.00$ & 0.384 & 1.89 & $0.59 \sim 6.25$ & 0.283 \\
\hline ongoing pregnancy & 0.85 & $0.45 \sim 1.59$ & 0.615 & 0.85 & $0.42 \sim 1.69$ & 0.639 \\
\hline
\end{tabular}

Effect of trigger protocol was evaluated as risk ratio(95\% $\mathrm{Cl})$ with dual trigger protocol as the reference.

† Adjusted for female age, BMI, primary infertility, infertility duration, embryo stage and endometrium thickness.

‡Poisson model was used to estimate OR when GEE log binomial models did not converge.

\section{Discussion}

This retrospective analysis is the first to employ a robust statistical model (cluster-weighted GEE model) tocompare the clinical efficiency of commonly used trigger protocols, hCG and dual trigger, in poor ovarian responders. Our results showed that dual trigger might not have any superioritiesoverhCG trigger in terms of oocyte retrieval cancellation, cycles rate of oocyte harvesting, and number of retrieved oocytes, mature oocyte, fertilized eggs, viable embryos as well top-quality embryos. Furthermore, dual trigger didn't improve the outcome of embryo transfer yet囚including implantation, clinical pregnancy or early miscarriage. Our results indicated that addition of $\mathrm{GnRHa}$ to the trigger regimen cannotbetter the outcome of IVF-ETfor DOR patients.

Due to the structural similarity to LH (sharing the same a subunit and $85 \%$ of the amino acid sequence of the $\beta$ subunit), and consequent vigorous binding and activating of LH/HCG receptor[2], hCGhas beenroutinelyused to induce oocyte maturation and ovulation for several decades in artificial reproductive treatment.With a stronger affinity $(6 \sim 8$ times that of $\mathrm{LH})$, and longer half-life,itdisplays a violent power intriggering oocyte maturation, but concurrently, raises a higher risk of OHSS as well as impaired embryo quality $[6,19]$. By binding to pituitary $\mathrm{GnRH}$ receptor and exerting a "flare up" role,GnRHa caninduce an endogenous natural-like mid-cycle hormone surge (including LH peak and FSH secretion) [3].In view of thebiological role of $\mathrm{FSH}$ in regulation of $\mathrm{LH}$ receptor expression, one potential benefit proposed for $\mathrm{GnRHa}$ or dual trigger is improved maturation and quality of oocytes, and subsequently, more viable embryos [20-23]. This improvement has been extensively checked in high responders[7, 24, 25],normal responders $[9,10,26,27]$, and poor responder[11-14], however, the current viewpoints remain controversy.Despite several prospective studies showed GnRHa trigger result in a higher number and percentage of mature oocytes as compared with hCG[24,28], a pooled analysis including four eligible random controlled trials failed to show any difference in the number of either retrieved oocytes, or mature 
oocytes[29].Thus, it is not unexpected that our study did not identify any significance in oocyte retrieval or oocyte maturation between the two triggers.

From the biological basis, it isunilateral to consider an indisputable superiority for $\mathrm{GnRHa}$ in triggering oocyte maturation.Indeed,GnRHa can induce an endogenous mid-cycle FSH surge, whichmightendow it with an advantage in cumulus expansionand consequent oocyte maturation, whereas thepeak time,duration, and profile oftheinduced gonadotrophins secretionisdistinct from that of the natural cycle[4, 30]. For these non-physiological features, we should furtherly considercertain unfavourability ofGnRHawhen using it to trigger the oocyte maturation process.It is widely acknowledged that, under COS conditions, poor oocyte quality and embryo development partly lie in a stubborn asynchrony between nuclear and cytoplasmic maturationprocess of oocytes[31, 32]. Despite the explicit underlying mechanism underlying this asynchrony remainselusive, a premature resumption of oocyte meiosis induced byexogenous pharmacological drugs, including GnRHaor HCGmaypresumablyplay an important role.Basic researches have indicated that the transportation of signal molecules, nutrients, and energy substrates through the gap junction, from cumulus cell to oocytes, be requisite for not onlyoocyte nuclear maturation, but also its cytoplasmic maturation $[33,34]$.In respond to the LH surge and its downstream ovulatory signals, such as EGF-like peptides (including AREG, EREG and BTC), EGFR-ERK1/2 as well as NPPC-NPR1 signal pathways[35-37], a diminished phosphorylation of Cx43 and a consequential closure of gap junction between oocytes and cumulus cells will emerge in concurrence with the meiosis resumption and progress, i.e., nuclear maturation[38-40]. In terms of the pharmacokinetics, the peak time of hCGafter administration is about $12 \mathrm{~h}$ [41], while the LH peak induced by GnRHaonly $4 \mathrm{~h}[30$ ], which is evenprecocious in relation to thenatural cycles. This relative premature LH surge may induce anuntimely interruption of the junction between oocytes and cumulus cells, which in turn, eliminate the accumulation of oocyte quality factors such as signal molecules, nutrients, and energy substrates, and eventuallyaggravate the asynchrony between nuclear and cytoplasmic maturation. On the other way,cumulus granulosa cell expansion induced by mid-cycle FSH surge also mechanically destroys the gap junctions[42]. Another concernforGnRHa trigger lies in thereduced amount of released gonadotrophins. The area under the gonadotrophins curve (AUC) after $\mathrm{GnRHa}$ administration has been reported to be significantly decreased as compared with that in natural cycle, or HCG[4], which raises the risk of insufficient maturity trigger. Thus, a preconceived proposal that $\mathrm{GnRHa}$ or dual trigger should acquire to a superior outcome still lack solid biological basis.All these complexities in the biological roles of GnRHa trigger may partly explained the contradictory conclusion of current works. Our study, together with others, suggest that the biological advantages of dual trigger in theory may be of no clinical significance for DOR patients[13,43]. Our additional analysis with the embryo transfer dataset yet failed

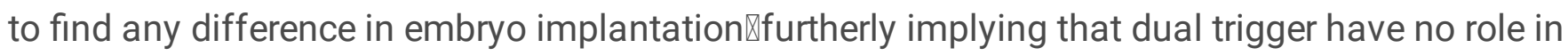
improving the oocyte quality.

One divergence of our analysis from others was embodied in the diversity of ovarian stimulating protocols, including mini-stimulation, PPOS, and GnRH-antagonist protocols. Inclusion of single COS protocol, the way of most previous studies,seems to be partly driven by the consideration that COS protocols may impose an essential impact on the clinical outcomes such as oocyte retrieval, oocyte 
maturation, fertilization, embryo development and implantation. Indeed, the COS protocols distributed unevenly between the two groups. Nevertheless, the analysis focusing on the marginal effect demonstrated that COS protocols do not affect either cultural or implantation outcomes [data not shown]. Then the diversity in COS may not impair the robustness of present study, and furtherly strengthen the extrapolation for our conclusion.

When choosing anappropriate model for statistical analysis, we should consider certain tricky features of the IVF-ET data. Firstly, repeated observation at the levels of cycles, oocytes or embryos lead to an evident correlation withinthese observation objectives, making the data usually deviating from normal distribution or lognormal distribution. Thisdatatype is beyond the reach of traditional statistical methods, such as univariate model, logistic or multiple linear regressions[44]. Secondly, the cluster size, i.e., the number of retrieved oocytes, embryos, or repeated cyclesof individuals in IVF practice, has been universally acknowledged to be informative for the clinical outcome, for example, the count of repeated cycles may contribute to the varianceof response variables, such as implantation rate and top-quality embyos. Henceforth,acluster-weighted statistical model considering the nonignorable cluster size is necessary in this context, for example, cluster-weighted GEE ormixed effect models.Finally, some confounding factors, such as age andavailable follicles, have so tremendous an influence on reproductive outcomes that evenslight baselineunevenness (despite no significance) may give rise to apoor comparison between studied groups. This covariates should be adjusted in a multivariate analysis model[45]. Nevertheless, most of the current studies have not fully considered above factors, and mightgive unrobust,and even inconsistent conclusions. In present study, we exploited a cluster-weighted GEE model to evaluate the marginal effects of trigger protocols and related covariates, which is suitable for multivariate analysis of non-normal, repeated observational or cluster data, and might produce a considerably reliable result.

Taken together, from present study, we preliminarily believe that dual trigger be not superior to conventional hCG protocol in term of either embryo outcomes or implantation outcomes during IVF-ET for DOR patients. However, because this is merely a retrospective investigation, further large-sample, prospective research is expected to provide a more solid conclusion.

\section{Conclusions}

Dual triggering the final oocyte maturation combined of $\mathrm{GnRH}-\mathrm{a}$ and standard dose of hCG cannot significantly improve the retrieval, cultural or implantation outcomes of IVF-ET treatments in women with DOR.

\section{Abbreviations}

DOR囚diminished ovarian reserve

$\cos \bowtie$ controlled ovarian stimulation 
FET囚frozen embryo transfer

IVF-ET囚in vitro fertilization and embryo transfer

hCG『human chorionic gonadotropin

GnRHa囚gonadotropin releasing hormone agonist

LH囚luteinizing hormone

FSH®follicle-stimulating hormone

WGGE\weighted generalized estimating equation

$\mathrm{BMI}, \bigotimes$ body mass index;

AMH囚anti-Mullerian hormone

AFC囚antral follicle count

hMG囚human menopausal gonadotropin

PPOS『progestin-primed ovarian stimulation

MPA囚medroxyprogesterone acetate

COCs\cumulus-oocyte complexes

ICSI囚intracytoplasmic sperm injection

HRT \hormone replacement

OPU\oocyte pick-up

GEE囚generalized estimating equation

\section{Declarations}

Ethical Approval and Consent to participate

Not applicable

Consent for publication

Not applicable

Availability of supporting data 
The datasets used and/or analyze during the current study are available from the corresponding author on reasonable request.

\section{Competing interests}

The authors declare no competing interests in this study.

\section{Funding}

Not applicable

\section{Authors' contributions}

Ling Zhang, Li-mei Wu wrote the main manuscript text and Meng-xia Ji, Shi-shi Li, Lin Zhang, Zhen Jin, Yi-er Zhou, Chong-yi Shu prepared figures 1-6. Jing Shu and Wei-hai Xuachieved the assessment of the quality of the literature. All authors reviewed the manuscript.

\section{Acknowledgements}

This study was supported 'New Century 151 Talent Program' of Zhejiang Province (L.Z)

\section{References}

1. Ubaldi, F., et al., Management of Poor Responders in IVF: Is There Anything New? BioMed Research International, 2014. 2014: p. 352098.

2. Kessler, M., et al., Structure of N-glycosidic carbohydrate units of human chorionic gonadotropin. The Journal of biological chemistry, 1979. 254: p. 7901-8.

3. Dosouto, C., T. Haahr, and P. Humaidan, Gonadotropin-releasing hormone agonist (GnRHa) trigger State of the art. Reprod Biol, 2017. 17(1): p. 1-8.

4. Humaidan, P., et al., GnRH agonist for triggering of final oocyte maturation: time for a change of practice? Hum Reprod Update, 2011. 17(4): p. 510-24.

5. Ortega, I., J.A. García-Velasco, and A. Pellicer, Ovarian manipulation in ART: going beyond physiological standards to provide best clinical outcomes. Journal of assisted reproduction and genetics, 2018. 35(10): p. 1751-1762.

6. Engmann, L., C. Benadiva, and P. Humaidan, GnRH agonist trigger for the induction of oocyte maturation in GnRH antagonist IVF cycles: a SWOT analysis. Reproductive BioMedicine Online, 2016. 32(3): p. 274-285.

7. Li, S., et al. Dual trigger of triptorelin and HCG optimizes clinical outcome for high ovarian responder in GnRH-antagonist protocols. Oncotarget, 2018. 9, 5337-5343 DOI: 10.18632/oncotarget.23916.

8. Orvieto, R., Triggering final follicular maturation- $h C G$, GnRH-agonist or both, when and to whom? Journal of Ovarian Research, 2015. 8(1): p. 60.

9. Haas, J., et al., GnRH agonist and hCG (dual trigger) versus hCG trigger for final follicular maturation: a double-blinded, randomized controlled study. Human Reproduction, 2020. 35(7): p. 1648-1654. 
10. Lin, M.-H., et al., Dual trigger with combination of gonadotropin-releasing hormone agonist and human chorionic gonadotropin significantly improves the live-birth rate for normal responders in GnRH-antagonist cycles. Fertility and Sterility, 2013. 100(5): p. 1296-1302.

11. Mutlu, I., et al., Dual trigger with the combination of gonadotropin-releasing hormone agonist and standard dose of human chorionic gonadotropin improves in vitro fertilisation outcomes in poor ovarian responders. Journal of Obstetrics and Gynaecology, 2021: p. 1-6.

12. Chern, C.-U., et al., Dual-trigger improves the outcomes of in vitro fertilization cycles in older patients with diminished ovarian reserve: A retrospective cohort study. PloS one, 2020. 15(7): p. e0235707e0235707.

13. Lin, M.-H., et al., Dual trigger with gonadotropin releasing hormone agonist and human chorionic gonadotropin significantly improves live birth rate for women with diminished ovarian reserve. Reproductive Biology and Endocrinology, 2019. 17(1): p. 7.

14. Zhang, J., et al., Dual trigger of final oocyte maturation in poor ovarian responders undergoing IVF/ICSI cycles. Reproductive BioMedicine Online, 2017. 35(6): p. 701-707.

15. Medicine, A.S.i.R. and E.S.I.G.o. Embryology, The Istanbul consensus workshop on embryo assessment: proceedings of an expert meetingt. Human Reproduction, 2011. 26(6): p. 1270-1283.

16. Gardner, D.K. and W.B. Schoolcraft, Culture and transfer of human blastocysts. Current Opinion in Obstetrics and Gynecology, 1999. 11(3).

17. Missmer, S.A., et al., Analysis of multiple-cycle data from couples undergoing in vitro fertilization: methodologic issues and statistical approaches. Epidemiology (Cambridge, Mass.), 2011. 22(4): p. 497-504.

18. Williamson, J.M., H.-Y. Kim, and L. Warner, Weighting Condom Use Data to Account for Nonignorable Cluster Size. Annals of Epidemiology, 2007. 17(8): p. 603-607.

19. Valbuena, D., et al., Increasing levels of estradiol are deleterious to embryonic implantation because they directly affect the embryo. Fertility and Sterility, 2001. 76(5): p. 962-968.

20. RICHARDS, J.S., et al., Ovarian Follicular Development in the Rat: Hormone Receptor Regulation by Estradiol, Follicle Stimulating Hormone and Luteinizing Hormone1. Endocrinology, 1976. 99(6): p. 1562-1570.

21. ZELEZNIK, A.J., A.R. MIDGLEY, JR, and L.E. REICHERT, JR, Granulosa Cell Maturation in the Rat: Increased Binding of Human Chorionic Gonadotropin Following Treatment with Follicle-Stimulating Hormone in Vivo11. Endocrinology, 1974. 95(3): p. 818-825.

22. Eppig, J.J., FSH stimulates hyaluronic acid synthesis by oocyte-cumulus cell complexes from mouse preovulatory follicles. Nature, 1979. 281(5731): p. 483-484.

23. Strickland, S. and W.H. Beers, Studies on the role of plasminogen activator in ovulation. In vitro response of granulosa cells to gonadotropins, cyclic nucleotides, and prostaglandins. Journal of Biological Chemistry, 1976. 251(18): p. 5694-5702.

24. Humaidan, P., et al., GnRH agonist (buserelin) or hCG for ovulation induction in GnRH antagonist IVF/ICSI cycles: a prospective randomized study. Human Reproduction, 2005. 20(5): p. 1213-1220. 
25. Griffin, D., et al., Dual trigger of oocyte maturation with gonadotropin-releasing hormone agonist and low-dose human chorionic gonadotropin to optimize live birth rates in high responders. Fertility and sterility, 2012. 97(6): p. 1316-1320.

26. Gao, F., et al., Effect of a "Dual Trigger" Using a GnRH Agonist and hCG on the Cumulative Live-Birth Rate for Normal Responders in GnRH-Antagonist Cycles. Frontiers in Medicine, 2021. 8(759).

27. Zhang, Y., et al., Outcomes comparison of IVF/ICSI among different trigger methods for final oocyte maturation: A systematic review and meta-analysis. The FASEB Journal, 2021. 35(7): p. e21696.

28. Oktay, K., I. Türkçüoğlu, and K.A. Rodriguez-Wallberg, GnRH agonist trigger for women with breast cancer undergoing fertility preservation by aromatase inhibitor/FSH stimulation. Reproductive BioMedicine Online, 2010. 20(6): p. 783-788.

29. Griesinger, G., et al., GnRH agonist for triggering final oocyte maturation in the GnRH antagonist ovarian hyperstimulation protocol: a systematic review and meta-analysis. Human reproduction update, 2006. 12(2): p. 159-168.

30. Itskovitz, J., et al., Induction of preovulatory luteinizing hormone surge and prevention of ovarian hyperstimulation syndrome by gonadotropin-releasing hormone agonist**Presented in part at the International Symposium on GnRH Analogues in Cancer and Human Reproduction, Geneva, Switzerland, February 18 to 21, 1988, and at the 6th World Congress of In Vitro Fertilization and Alternative Assisted Reproduction, Jerusalem, Israel, April 2 to 7, 1989. Fertility and Sterility, 1991. 56(2): p. 213-220.

31. Sundström, P. and B.O. Nilsson, Meiotic and cytoplasmic maturation of oocytes collected in stimulated cycles is asynchronous. Human Reproduction, 1988. 3(5): p. 613-619.

32. Pereira, N., et al., The role of in-vivo and in-vitro maturation time on ooplasmic dysmaturity. Reproductive BioMedicine Online, 2016. 32(4): p. 401-406.

33. Carabatsos, M.J., et al., Oocyte-Granulosa Cell Heterologous Gap Junctions Are Required for the Coordination of Nuclear and Cytoplasmic Meiotic Competence. Developmental Biology, 2000. 226(2): p. 167-179.

34. Landschaft, D., Gaps and barriers: Gap junctions as a channel of communication between the soma and the germline. Seminars in Cell \& Developmental Biology, 2020. 97: p. 167-171.

35. Park, J.-Y., et al., EGF-Like Growth Factors As Mediators of LH Action in the Ovulatory Follicle. Science, 2004. 303(5658): p. 682-684.

36. Zhang, M., et al., Granulosa Cell Ligand NPPC and Its Receptor NPR2 Maintain Meiotic Arrest in Mouse Oocytes. Science, 2010. 330(6002): p. 366-369.

37. Xi, G., et al., The mRNA-destabilizing protein Tristetraprolin targets "meiosis arrester" \&lt;em\&gt;Nppc\&/t;/em\&gt; mRNA in mammalian preovulatory follicles. Proceedings of the National Academy of Sciences, 2021. 118(22): p. e2018345118.

38. Granot, I. and N. Dekel, Phosphorylation and expression of connexin-43 ovarian gap junction protein are regulated by luteinizing hormone. Journal of Biological Chemistry, 1994. 269(48): p. $30502-$ 30509. 
39. Cai, $\mathrm{H} .$, et al., Involvement of PKCE in FSH-induced connexin43 phosphorylation and oocyte maturation in mouse. Biology Open, 2018. 7(8).

40. Sela-Abramovich, S., et al., Mitogen-Activated Protein Kinase Mediates Luteinizing Hormone-Induced Breakdown of Communication and Oocyte Maturation in Rat Ovarian Follicles. Endocrinology, 2005. 146(3): p. 1236-1244.

41. Chan, C.C.W., et al., Bioavailability of $h C G$ after intramuscular or subcutaneous injection in obese and non-obese women. Human Reproduction, 2003. 18(11): p. 2294-2297.

42. Atef, A., et al., The potential role of gap junction communication between cumulus cells and bovine oocytes during in vitro maturation. Molecular Reproduction and Development, 2005. 71(3): p. 358367.

43. Eftekhar, M., et al., A comparison of dual triggering (by administration of GnRH agonist plus HCG) versus HCG alone in poor ovarian responders in ART outcomes. Middle East Fertility Society Journal, 2018. 23(4): p. 350-353.

44. Chiu, Y.H., et al., Serum omega-3 fatty acids and treatment outcomes among women undergoing assisted reproduction. Human reproduction (Oxford, England), 2018. 33(1): p. 156-165.

45. Farquhar, C.M., et al., Female subfertility. Nature Reviews Disease Primers, 2019. 5(1): p. 7. 